\title{
Sources of variability in interceptive movements
}

\author{
Eli Brenner · Jeroen B. J. Smeets
}

Received: 24 September 2008/ Accepted: 24 February 2009/Published online: 13 March 2009

(C) The Author(s) 2009. This article is published with open access at Springerlink.com

\begin{abstract}
In order to successfully intercept a moving target one must be at the right place at the right time. But simply being there is seldom enough. One usually needs to make contact in a certain manner, for instance to hit the target in a certain direction. How this is best achieved depends on the exact task, but to get an idea of what factors may limit performance we asked people to hit a moving virtual disk through a virtual goal, and analysed the spatial and temporal variability in the way in which they did so. We estimated that for our task the standard deviations in timing and spatial accuracy are about $20 \mathrm{~ms}$ and $5 \mathrm{~mm}$. Additional variability arises from individual movements being planned slightly differently and being adjusted during execution. We argue that the way that our subjects moved was precisely tailored to the task demands, and that the movement accuracy is not only limited by the muscles and their activation, but also- and probably even mainlyby the resolution of visual perception.
\end{abstract}

Keywords Interception - Optimal · Arm movements · Timing · Accuracy · Planning · Vision · Eye movements · Human

\section{Introduction}

There are many sources of variability in human goaldirected movements (van Beers 2007), and it seems safe to assume that people will try to move in a way that will

E. Brenner $(\bowtie)$ J. B. J. Smeets

Faculty of Human Movement Sciences, Vrije Universiteit,

Van der Boechorststraat 9,

1081 BT Amsterdam, The Netherlands

e-mail: e.brenner@fbw.vu.nl minimize the detrimental effects of such variability (van Beers 2008; Brenner and Smeets 2007; Harris and Wolpert 1998; Müller and Sternad 2004; Trommershauser et al. 2003). An obvious way to do so is by minimizing the variability itself, for instance by moving slowly. It is clear that faster movements are less accurate, even if it is not clear exactly why, with explanations varying from signaldependent noise in the neuronal signals sent to the muscles (Harris and Wolpert 1998) to the time available for visual guidance (Fernandez and Bootsma 2004). Apart from trying to reduce the variability by moving slowly, one could also try to reduce the impact of variability, for instance by approaching surfaces orthogonally (Brenner and Smeets 1995). However, to do so one may need to move along a more curved path, and moving on a curved path increases variability, so a compromise has to be found (Brenner and Smeets 2007).

When interacting with moving objects there is the additional complication that in order to move more slowly one must either start moving earlier or move to a different place. Moreover, although moving slowly reduces the spatial variability, it increases the temporal variability, so again a compromise must be found (Brouwer et al. 2000). Furthermore in many cases the task is not simply to make contact with the moving object. If the task is for instance to hit a ball as far as possible, a compromise may need to be found between hitting the ball on many trials but not quite as far as one could, and hitting it on fewer trials but further. Other factors such as energy expenditure may also be considered, especially if one has to repeat the movement many times. Thus, it should be clear that there is more to tasks involving interception than just reaching some point at the right time.

Variability between interceptive movements undoubtedly arises at many stages; from deciding what kind of 
movement will best realize the ultimate goal of the interception, to changes in how the muscles respond to the signals that drive them (e.g., through fatigue). Inaccuracy of sensory information about the target or about ones own posture could obviously also introduce variability, both when the movement is planned and when previous errors are corrected on the basis of updated sensory information. Considering recent experience, both in terms of the properties of the target and in terms of the success or failure on previous trials, can also influence the variability (de Lussanet et al. 2001), for instance by providing more robust estimates of target properties or by enticing one to reevaluate the importance of various constraints. In the present study we try to shed some light on the contributions of various sources to the variability of goal-directed movements. We use an interception task that requires high spatial and temporal accuracy. By analysing the variability between repeated trials, in particular at the moment of impact, we try to separate errors with a spatial from ones with a temporal origin, and to determine what strategic decisions are made. We then try to relate these decisions to possible sensory and motor causes of variability.

\section{Methods}

We conducted a single experiment in which subjects had to hit a simulated disk into a goal. Subjects had full vision of their hand, the disk and the goal, and both the movements of their hand and of their eyes were recorded. Subjects could also see the disk move in its new direction after they hit it, and received explicit feedback about whether they had 'scored'.

\section{Equipment}

Figure 1 is a schematic depiction of our setup. Images were projected from above onto a back-projection screen, $20 \mathrm{~cm}$ above a half-silvered mirror. There was a large (WACOM A2) drawing tablet $20 \mathrm{~cm}$ below the mirror, positioned so that it coincided precisely with the apparent position of the screen as seen through the mirror. Lamps between the halfsilvered mirror and the drawing tablet (not shown) ensured that subjects could clearly see the stylus and their hand as well as the disk. A simple calibration whereby the experimenter aligned the tip of the stylus with small disks presented on the screen allowed us to later relate any position in the image to a position on the surface of the drawing tablet, and vice versa. Images were generated at $85 \mathrm{~Hz}$ with a resolution of 1024 by 768 pixels. The experiments were conducted in a normally illuminated room.

Subjects intercepted the moving disks by moving a stylus across the drawing tablet. The stylus looked and felt

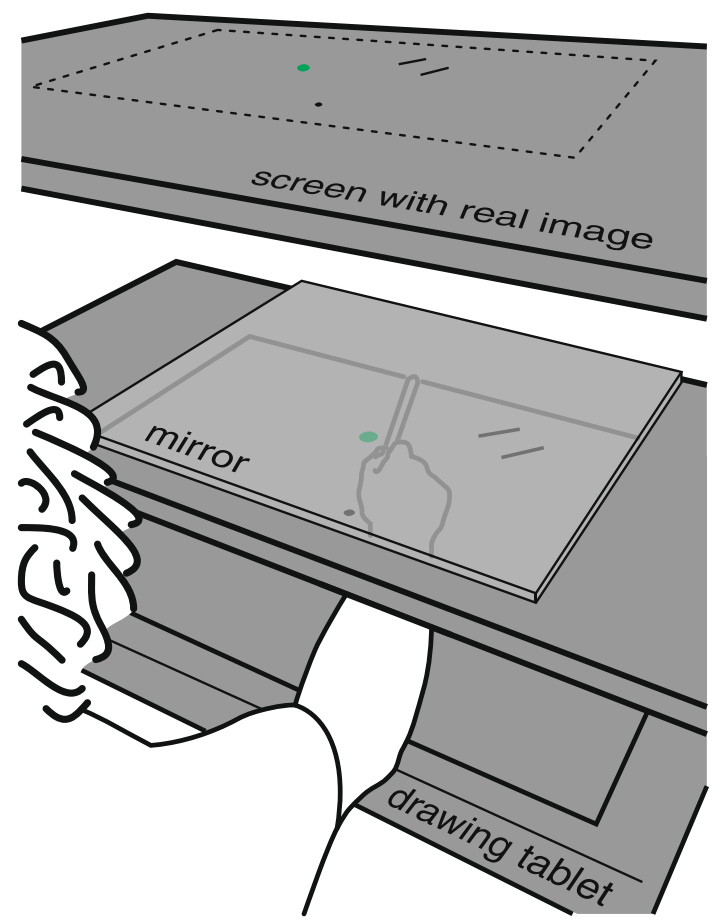

Fig. 1 The setup. Subjects moved the stylus that they held in their hand across the surface of the drawing tablet to hit a moving disk into a goal. They saw their hand through the half-silvered mirror. The starting point, disk and goal were projected from above onto the screen above the mirror, but appeared to be on the tablet

like a normal pen, and was held like a normal pen, but it did not leave any trace when it was moved. Instead, the tablet determined the position of its tip at $200 \mathrm{~Hz}$. There were obviously delays in our equipment, especially within the graphics tablet and when presenting the images. In the analysis we corrected for all such delays. However, as a result of the delays the disk continued to move along its original path for about $62 \mathrm{~ms}$ after being hit. At that time the disk suddenly jumped from a position that it should never have reached to the appropriate position on the new path, and then continued along that path. Subjects did not notice this, perhaps because the hand passed through the disk during that time. The delays were considered when determining the disk's new path, so except for the abovementioned overshoot all feedback was correct.

We also measured the subjects' eye and head movements. The movements of the eyes relative to the head were recorded at $500 \mathrm{~Hz}$ using an Eyelink II (SR Research Ltd, Canada). The orientations of the two eyes were averaged to estimate a single orientation of gaze relative to the head. Head movements were recorded at $250 \mathrm{~Hz}$ using the head tracking capabilities of the Eyelink (and converted into head position and orientation using custom software). We determined where on the surface of the tablet the subject was looking from the position and orientation of the head and the orientation of gaze relative to the head. 
Properties of the disk and goal

The task involved a disk and a goal. The disk was green and had a diameter of $3 \mathrm{~cm}$. In terms of its simulated physical properties it had no friction or air resistance and a negligible mass compared with the stylus. Thus it moved at a constant velocity until impact, and then continued at a new constant velocity after impact, where the new motion was the vector sum of the old one and twice the velocity of impact. The velocity of impact is defined as the component of the velocity of the stylus relative to the disk (at impact) that is perpendicular to the disk's edge at the point of contact.

The goal consisted of two parallel $8 \mathrm{~cm}$ lines that were $8 \mathrm{~cm}$ apart. The lines were either oriented $45^{\circ}$ or $-45^{\circ}$ with respect to the sagittal direction. Figure $2 \mathrm{a}$ shows the two possible goal locations (drawn to scale). When the disk was straight in front of the starting point (intersection between the dashed line and the vertical dotted line) it was also straight in front of the goal (dotted diagonal lines). The center of the disk was then $10 \mathrm{~cm}$ from the center of the goal. The task was to hit the disk so that it passed between the two lines.

\section{Conditions}

There were four conditions that only differed in the direction in which the disk was moving (to the left or to the right), the position at which the disk appeared $(13.66 \mathrm{~cm}$ to the right or left), and the position and orientation of the goal. The disk always moved at the same speed $(20 \mathrm{~cm} / \mathrm{s})$ and was always straight in front of the starting point $683 \mathrm{~ms}$ after it started moving. Figure $2 \mathrm{~b}$ shows a schematic representation of the four combinations of goal position and disk motion.

\section{Subjects and procedure}

Nine subjects took part in the experiment, including one of the authors. Each subject took part in a single session of 200 trials (50 for each of the four conditions). The session started with a simple calibration of the Eyelink system, whereby the subject rotated his or her head for some time while fixating a point at the screen center. The calibration terminated when the subject had made 20 head movements for which the eye would have had to rotate at least $5^{\circ}$ to maintain fixation. From the measured position and orientation of the head (relative to the screen center) we calculated the orientations of the eyes in the head at each moment (assuming that the subject maintained fixation). We used these orientations to calibrate the cameras of the Eyelink system, relating camera positions of the pupil to orientations of the eyes. This is a less accurate method than

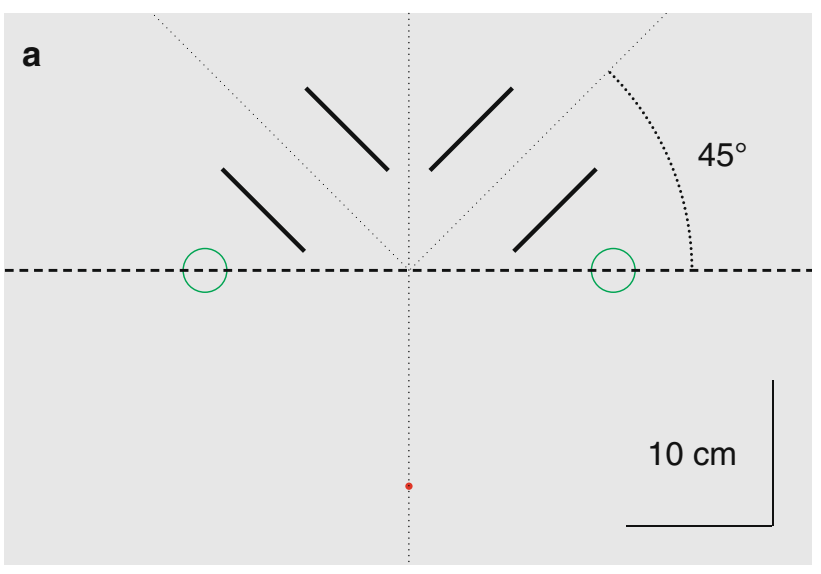

b

\section{disk moving to the}

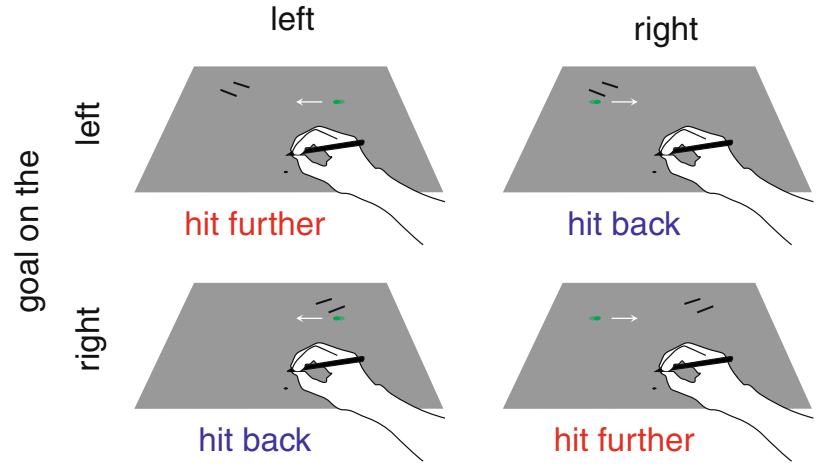

Fig. 2 The layout of the task. a The disk moved laterally (path shown by dashed line) from one of two starting positions (represented by green circles) at $20 \mathrm{~cm} / \mathrm{s}$. A trial always started with the stylus at the starting position (red dot). Only one goal (pairs of thick lines) and one disk were present on each trial. b The four conditions. The disk either moved to the left or to the right and the goal was either on the left or on the right. In all cases the disk had to deviate from its path to pass between the lines of the goal, but in two cases the deviation was modest (about $45^{\circ}$; hit further) whereas in the other two the deviation was large (about $135^{\circ}$; hit back). In the latter cases the disk occluded $1.2 \mathrm{~mm}$ of the closest line of the goal as it passed, but this was not disturbing

the standard Eyelink calibration, but it is fast and circumvents the problem that the standard Eyelink calibration assumes that the image is on a more or less frontally placed screen. After eye movement calibration we explained the task and subjects could practice as often as they liked while the experimenter checked that the eye and head movements could be recorded throughout the movement. As soon as the subject was confident with the task the actual recording session began. During the session the 200 trials were presented in random order.

Each trial started with a $5 \mathrm{~mm}$ diameter red dot appearing at a fixed position on the screen, close to the subject. Subjects started a trial by moving the tip of the stylus to this starting point and holding it still. The starting point disappeared shortly after the subject placed the stylus 
within its bounds. At that moment the green $3 \mathrm{~cm}$ diameter disk and the goal appeared. The disk moved laterally, along a line that was $15 \mathrm{~cm}$ further from the subject than the starting point (dashed line in Fig. 2a). The subjects' task was to hit the disk so that it would move between the two bars that formed the goal, without it touching either of them. We considered the disk to have been hit as soon as the (interpolated path of the) stylus made contact with the disk's contour (at some interpolated position). Once the stylus hit the disk the latter moved on in accordance with the simulated properties described above. If the disk passed successfully between the goal lines, subjects heard a short sound and the goal temporarily turned yellow.

\section{Analysis}

The first parameter that we examined was obviously how successful subjects were at fulfilling the task. On the basis of this (see Results) we decided to exclude the first 20 trials from the further analysis. Whenever possible we analysed all remaining 180 trials, irrespective of whether the disk was hit and whether it passed through the goal. Some measures obviously only exist if the disk was hit, such as the direction in which it moved after being hit, or where on its surface the stylus hit it. When it is not obvious whether only trials in which the stylus did hit the disk could be considered this is mentioned explicitly. To get a general impression of the movements we determined the average reaction time (time between the disk appearing and the stylus moving $5 \mathrm{~mm}$ from where it was when the disk appeared), the average movement time (time between the stylus having moved $5 \mathrm{~mm}$ and it making contact with the surface of the disk; obviously only for trials in which the disk was hit), the average path and the average velocity profile.

The average path was calculated by first re-sampling the lateral position of the stylus on each trial for 100 equal $1.5 \mathrm{~mm}$ steps in depth (i.e., in the sagittal direction), from the starting point until the stylus crossed the disk's path. The re-sampling consisted of first estimating the time that the stylus reached the distance that corresponds to the step in question, then fitting a straight line to all samples between $20 \mathrm{~ms}$ before and $20 \mathrm{~ms}$ after that moment, and finally estimating the lateral position of this line at the relevant distance. This procedure provides a slightly smoothed interpolation. The estimated lateral positions were then averaged for each subject and condition. Using the distance of the disk's path as the endpoint, rather than contact with the disk, allows us to include trials in which the stylus missed the disk, and has the advantage that we can be sure that anything that we see in the path is not an artifact of selecting trials that turned out to be successful. The stylus' average tangential velocity profile was sampled for the same 100 steps in depth. We took the distance between the measured stylus positions just before and just after the stylus passed the distance that corresponds to the step in question, and divided this by the $5 \mathrm{~ms}$ between the samples to obtain an unsmoothed estimate of velocity. This procedure allows us to calculate average velocities at each position along the path.

Since the impact between the stylus and the disk is critical for succeeding in the task we analysed performance near this time in more detail. To do so we determined the values of several parameters near the moment of impact. Three parameters were determined for trials in which the disk was hit: the position of the disk at the moment that it was hit, the position on the disks' surface at which the stylus made contact, and the direction in which the disk moved after being hit. These values were based on interpolation in the same manner as described for the path above, but now for the lateral and sagittal positions as a function of time. For four additional parameters we did not use the moment of impact itself, but (an estimate of) the planned moment of impact. This was defined as the moment the stylus reached the average (sagittal) distance at which it was when it did actually hit the disk. Separate average distances were determined for trials in which the disk was hit further and back because the appropriate distance is different in these two cases (due to the symmetrical geometry of the task we did not determine separate distances for the two directions of disk motion). The distances were obviously only based on trials in which the disk was hit. The advantage of using the moment that the stylus reached a fixed distance, rather than the actual impact with the disk, is that the four parameters in question can be determined independently of whether and how the disk was hit. The four parameters that were determined in this manner are the stylus' lateral position, the direction and speed of its motion, and the disk's position. From the first and the last parameter we also calculated the position of the stylus relative to the disk at the planned moment of impact (for each trial). Finally we also determined the lateral position of the stylus when it was half way to the disk.

Average values and standard deviations were always calculated separately for each subject and condition. The average values and standard deviations were then averaged across the two mirror-symmetric conditions to provide one value per subject for hitting further and another for hitting back. We are particularly interested in the standard deviations because we use them to estimate the spatial and temporal resolution of task performance (as explained below). Besides comparing the above-mentioned measures we also determined the correlations between several of the above-mentioned parameters. These correlations were also calculated separately for each subject and condition, and then averaged. 
Distinguishing between variability in the plan and execution errors

As already mentioned, the critical moment in terms of task performance is when the stylus hits the disk. We therefore decided to take a closer look at the movement near the moment of the hit, ignoring how variability in performance at that moment depends on the movement until then. We initially expected subjects to always try to hit the disk when it was straight in front of the goal (at the intersection of the dashed and dotted lines in Fig. 2a), but it was immediately clear from the data that this was not the case. The variability in the position of both the stylus and the disk at the planned moment of impact was larger than the variability in their relative positions, so subjects could not even have always planned to hit the disk at the same time and place. We will therefore distinguish between what we call variability in the plan and execution errors. The basis for this distinction is that the task imposes certain correlations between when and how the disk should be hit, so any variability in the plan will adhere to these correlations, whereas random variability will not. The most obvious correlation is that between the positions of the stylus and the disk. Note that concentrating on the (planned) moment of impact implies that what we call variability in the plan is only 'planned' in terms of when and how the stylus moves when it reaches the disk. We do not distinguish between planning that occurs before the movement starts and changes to the plan while executing the movement. We also do not distinguish between variability that arises when judging the disk's position and velocity, or when judging the positions of the stylus and goal, and variability that arises when computing the optimal movement on the basis of such information. Thus, only uncorrected execution errors are not considered to be part of the planned variability.

We realize that planning is often considered to end at the moment the arm begins to move (although it is not at all evident that the way that movements are controlled changes fundamentally at that moment) while corrections to the arm movements are often attributed to feedback about the movement itself rather than to changes in the plan (although updates of the target position can be just as effective at guiding the hand; Brenner and Smeets 2003; Goodale et al. 1986). However, since we anyway have no way to tell how long in advance differences at the time of impact were planned, or whether they emerged from corrections to earlier errors, we will simply refer to both as being 'planned'. After evaluating the data in accordance with the above-mentioned distinction between variability in the plan and execution errors we will try to estimate to what extent the variability is planned before motion onset rather than being the consequence of corrections during the movement, and we will attempt to estimate the contribution of visual errors. But first we will use simulations based on this distinction to examine whether several measured aspects of our subjects' movements near the moment of impact are consistent with such a distinction.

\section{Simulation of task performance}

In each trial of our simulations we determined where subjects planned to hit the disk from a normal distribution with its peak at the average position that we found in the experiment and a standard deviation $\sigma_{\text {plan }}$ that we will estimate from the data in the manner described below. We assumed that subjects always planned to hit the disk through the center of the goal. Considering the average measured stylus velocity near the moment of impact, and the velocity of the disk, we could determine where along the disk's border the stylus would have to hit the disk in order for the disk to pass through the center of the goal (for each position of the disk when it was hit). In relation to this position, the hand's path in our simulations was sometimes too far to the left or to the right, and the hand was sometimes too early or too late. The average lateral position of the hand was correct, but on each trial there was an error drawn from a normal distribution with a standard deviation $\sigma_{\mathrm{s}}$. The average time at which the stylus reached the appropriate position for the planned hit was also correct, but again on each trial there was an error drawn from a normal distribution with a standard deviation $\sigma_{\mathrm{t}}$. Not moving exactly as planned implies that the stylus did not hit the disk at the planned moment, so we have to consider the direction and speed in which the stylus and disk are moving to determine the time and point at which the stylus hit the disk. In doing so we ignore the subtle changes in the stylus' velocity near the moment of impact, and use the average measured values of the speed and direction of motion at the moment of impact for the condition in question to determine when and where the stylus hit the disk (i.e., approximating the stylus' motion near the time of contact by motion at a constant speed along a straight line).

We estimated the magnitudes of the three kinds of variability that we used in our simulations $\left(\sigma_{\text {plan }}, \sigma_{\mathrm{s}}\right.$ and $\left.\sigma_{\mathrm{t}}\right)$ from the standard deviations in three of the measured parameters that were described above: the lateral position of the stylus when it reached the disk's path, both on the tablet $\left(\sigma_{\text {stylus }}\right)$ and relative to the disk $\left(\sigma_{\text {rel }}\right)$, and the time at which that the stylus reached the disk's path $\left(\sigma_{\text {time }}\right)$. By definition, errors are independent of the plan, so the variance in the measured lateral position of the stylus is a combination of the variance in the planned position at which the disk is hit and the spatial variance around the intended contact point: 
$\sigma_{\text {stylus }}^{2}=\sigma_{\text {plan }}^{2}+\sigma_{\mathrm{s}}^{2}$

Since planning to hit the disk at a different position also implies planning to hit it at a different time, a similar relationship holds for the variance in the moment that the stylus reaches the disk's path, which is a combination of the variance in the planned moment of contactconsidering the variability in the planned position at which the disk is hit-and the temporal variance around the intended moment of contact:

$\sigma_{\text {time }}^{2}=\left(\frac{\sigma_{\text {plan }}}{V_{\text {disk }}}\right)^{2}+\sigma_{\mathrm{t}}^{2}$

where $V_{\text {disk }}$ is the velocity of the disk. Equation 2 is an over-simplification because the planned position of contact on the disk's edge should depend on where the disk is when it is hit, whereas the equation only considers the planned position of the disk. However we ascertained that the contribution of changes in the position along the edge is negligible. Finally, since the variance in the position of the stylus relative to the disk is a combination of the spatial variance around the intended contact point and the influence of the temporal variance around the intended moment of contact (that again are considered to be independent):

$\sigma_{\text {rel }}^{2}=\sigma_{\mathrm{s}}^{2}+\sigma_{\mathrm{t}}^{2} V_{\mathrm{disk}}^{2}$

From Eqs. 1-3 and the measured values of $\sigma_{\text {stylus }}, \sigma_{\text {rel }}$, and $\sigma_{\text {time }}$ we can estimate the values of $\sigma_{\text {plan }}, \sigma_{\mathrm{s}}$, and $\sigma_{\mathrm{t}}$.

To determine the extent to which the geometry of the task and these three independent kinds of variability can explain our subjects' performance in the experiment, we simulated the outcome of 10,000 movements in which the disk was hit further and another 10,000 in which the disk was hit back. The simulations were conducted as described above, with the three randomly chosen values as the only source of variability between trials. Note that although we refer to $\sigma_{\mathrm{t}}$ as being temporal variability, this term actually includes a spatial component because misjudging the position along the stylus' path is indistinguishable from a timing error. In the discussion section we will try to separate these two kinds of error.

\section{Gaze}

Another way of trying to determine how subjects perform the task is by examining where they look while they do so. For instance, at each moment they are likely to direct their gaze toward the point at which they need the highest spatial resolution. We combined the measured eye and head movements to estimate where the subject was looking. We used a very small starting point so that subjects had to look at it before each trial in order to place the stylus adequately.
The calculated direction of gaze just before each trial was used to correct for drift in the eye movement recordings (assuming that all measured errors at that moment are due to such drift). We also used the (uncorrected) calculated direction of gaze at that moment to estimate the reliability of our measurements and calculations (assuming that the subject is fixating precisely). The average standard deviation in the position on the screen (for the 6 subjects for whom the eye movement recordings were successful) was $1.2 \mathrm{~cm}$ laterally and $1.8 \mathrm{~cm}$ in depth (the larger error in depth is due to the angle between the surface and the line of sight). We simultaneously smoothed and differentiated the gaze data by fitting a second order polynomial to the measured gaze position as a function of time for all data within $50 \mathrm{~ms}$ of each measured point in time (see Biegstraaten et al. 2006). This was done separately for the lateral and sagittal component.

At each point in time $(500 \mathrm{~Hz})$ we determined which 'objects' were within $5 \mathrm{~cm}$ of the calculated direction of gaze (stylus, disk, goal, starting point, point of interception). We also isolated the component of the change in the direction of gaze that was in the direction in which the disk was moving. If this component was between 0.4 and 2 times the velocity of the disk we considered the eyes to be pursuing the disk. Once the stylus had started moving we also isolated the component of the change in the direction of gaze that was in the direction in which the stylus was moving. If this component was between 0.4 and 2 times the velocity of the stylus at that moment we considered the eyes to be pursuing the stylus. Any gaze shifts that were not considered to be pursuit but that were faster than $20 \mathrm{~cm} / \mathrm{s}$ (which at this viewing distance and angle would correspond with about $20 \%$ s for horizontal motion and $15 \%$ for vertical motion) were considered to be part of a saccade. Slower motion was considered fixation. However, any such classification had to last for at least five samples $(10 \mathrm{~ms})$. Deviations from a classification that lasted only one sample were assigned to the neighboring class. Any measurement that did not fall into one of the above-mentioned classes was marked as uncertain, as did epochs characterized as pursuit but in which gaze did not appear to be directed within $5 \mathrm{~cm}$ of the object that was being pursued.

We distinguished between various kinds of saccades by examining their direction and near what they ended. Thus we could identify saccades toward any of the five objects mentioned above. We found some saccades that ended further than $5 \mathrm{~cm}$ from any object. These were classified as saccades to unspecified locations. We also found some saccades that ended within $5 \mathrm{~cm}$ of both the disk and the goal. If such saccades were followed by a saccade that landed within $5 \mathrm{~cm}$ of the goal but not of the disk, we considered the original saccade to have been toward the 
disk. If they were followed by a saccade that landed within $5 \mathrm{~cm}$ of the disk but not of the goal, we considered them to have been toward the goal. Periods of fixation were characterized by the object that was fixated, and if no object was within $5 \mathrm{~cm}$ of where the subject was looking we classified gaze as fixating an unspecified location.

Having characterized gaze in this manner we could analyse the sequences of eye movements. The eye movement data for trials in which gaze was directed at unspecified locations were excluded from the analysis because we assumed that they contained errors. This may not always be true because subjects may make saccades to positions at which they expect to see something that is not there. They may for instance sometimes make a fast saccade from the starting point to the left goal position, anticipating that it will appear there, while the goal actually appeared on the right. Since we do not really know why subjects made saccades to other locations than we expected, and they were certainly not all to positions such as the other possible goal position, we excluded trials with such saccades. To further simplify matters, horizontal saccades that were preceded and followed by pursuit were removed because they were considered to be catch-up saccades, as were short periods of fixation preceded and followed by pursuit (with no intervening saccade). If any data at all was missing within a trial (because the subject blinked or a head marker went out of view) the eye movement data for that trial was excluded from further analysis. The head camera quite frequently failed to register all four markers (actually two markers and their mirror images) because the markers had to be quite far apart to prevent them from getting in the way of the image (so that the space within which the camera could see all the markers was quite limited) and the subjects moved their heads quite a bit.

\section{Results}

One trial could not be used because the subject did not move at all. On 33 of the remaining 1799 trials the stylus missed the disk. These trials are included in the analysis of some measures, but not those that require that the disk be hit. The average reaction time was $266 \mathrm{~ms}$ when the disk was to be hit further and $260 \mathrm{~ms}$ when it was to be hit back (the $6 \mathrm{~ms}$ difference was not significant; paired $t$-tests across subjects). The corresponding average movement times were $466 \mathrm{~ms}$ and $479 \mathrm{~ms}$, respectively (difference not significant). Since the disk moved at $20 \mathrm{~cm} / \mathrm{s}$ and appeared $13.66 \mathrm{~cm}$ from the point from which it would move midway between the two lines that form the goal if it moved parallel to them after being hit (intersection of dotted lines in Fig. 2a), we may expect the sum of the reaction and movement times to be about $683 \mathrm{~ms}$. In fact subjects hit the disk about $50 \mathrm{~ms}$ later (i.e., when it was about $1 \mathrm{~cm}$ further; Table 1), perhaps because taking more time allowed them to be more accurate.

Figure 3 shows how successful subjects were at fulfilling the task of getting the disk through the goal. Performance fluctuated a bit, perhaps with a very gradual tendency to improve, but the differences were quite modest. Since performance may have been especially poor in the first two sets of 10 trials we decided to eliminate the first 20 trials from the further analysis. Perhaps we had not included quite enough training trials to achieve stable performance. Performance was also not equally good in all conditions. The best performance was found when the goal was on the left and the disk moved to the left (44.3\%), followed by the goal being on the right and disk moving to the right $(40.1 \%)$, then the goal on the left and disk motion to the right (39.3\%), and finally the goal on the right and disk motion to the left $(30.0 \%)$. Thus performance was

Table 1 Some measured values at the planned moment of impact

\begin{tabular}{lll}
\hline & Further & Back \\
\hline Average direction of stylus motion $(\mathrm{deg})$ & 35.7 & $32.3^{*}$ \\
Average velocity of stylus motion $(\mathrm{cm} / \mathrm{s})$ & 50.4 & $44.2^{* *}$ \\
Average position of disk $(\mathrm{cm})$ & 0.9 \\
Standard deviation in time to reach distance $\left[\sigma_{\text {time }}\right](\mathrm{ms})$ & 42 & $-1.2^{* * *}$ \\
Standard deviation in position of disk $\left[\sigma_{\text {disk }}\right](\mathrm{cm})$ & 0.84 \\
Standard deviation in position of stylus $\left[\sigma_{\text {stylus }}\right](\mathrm{cm})$ & 0.80 \\
Standard deviation in relative position $\left[\sigma_{\text {rel }}\right](\mathrm{cm})$ & 0.54 \\
Standard deviation in direction of stylus motion $(\mathrm{deg})$ & 7.59 \\
Standard deviation in tangential velocity of stylus $(\mathrm{cm} / \mathrm{s})$ & 13.6 \\
\hline Al vale & 0.96 \\
\hline
\end{tabular}

All values were calculated separately per subject and condition and then averaged. Separate values are shown for hitting the disk further and back. Relative position is the position of the stylus relative to the disk

Paired $t$-tests comparing subjects' average values when hitting further and back: * $P<0.05$; ** $P<0.01$; ** $P<0.001$ 


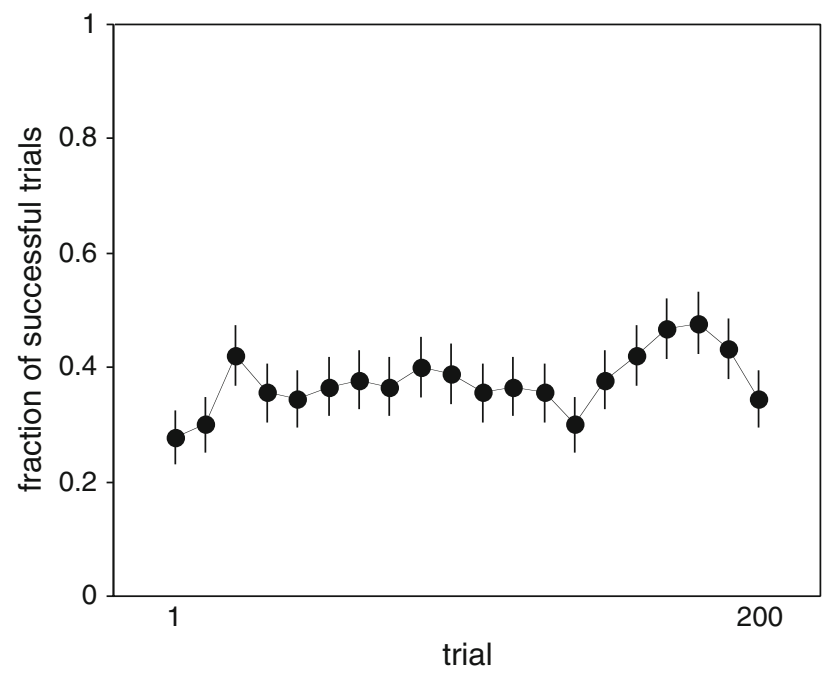

Fig. 3 Fraction of trials in which the disk passed through the goal as a function of the time course of the experiment. Each point is the average of the subjects' fractions of correct responses within bins of 10 consecutive trials (with the standard error across the nine subjects)

better when hitting further and when the goal was on the left.

Figure 4 shows the average paths (a) and velocity profiles (c) in the four conditions (b). As already mentioned, disks were generally hit after having crossed the midline. The stylus accelerated fast at the beginning of the movement and there was a small dip in the tangential velocity about three-quarters of the way to the disk, about at the time that the movement curved to approach the disk appropriately. The paths are more or less symmetrical, with the main distinction being between disks that have to be hit 'further' and 'back', so we will only distinguish between these two conditions in the remaining presentation, although all values were first determined for each of the four conditions separately and then averaged (as described in the Methods). To be able to simply average values across the two conditions in which the disk was to be hit either further or back, the movements when the goal was on the left were flipped laterally relative to the axis of symmetry (the sagittal line through the starting point; dotted vertical line in Fig. 2a). The resulting average paths are shown in Fig. 4d. The direction of stylus motion was defined relative to the sagittal direction, with positive angles indicating motion to the right. Similarly, lateral positions are defined relative to the sagittal line through the starting point, with positive values indicating positions to the right.

Table 1 shows various measured values. Our subjects cannot always have been aiming for the same position because the standard deviation in the stylus position relative to the disk $\left(\sigma_{\text {rel }}\right)$ is smaller than both the standard deviation in the stylus position $\left(\sigma_{\text {stylus }}\right)$ and that of the disk position $\left(\sigma_{\text {disk }}\right)$. Therefore the standard deviations in the positions of the disk and stylus, and in the time taken to reach the appropriate distance, combine influences of variations in the plan with spatial and temporal errors. We used Eqs. 1-3 and the average of the values for the two directions (further and back) of $\sigma_{\text {time }}, \sigma_{\text {stylus, }}$ and $\sigma_{\text {rel }}$ (45 ms, $0.9 \mathrm{~cm}$ and $0.62 \mathrm{~cm}$, respectively) to estimate the variability in the plan $\left(\sigma_{\text {plan }}\right)$ and the spatial and temporal errors $\left(\sigma_{\mathrm{s}}\right.$ and $\sigma_{\mathrm{t}}$; see Table 2$)$. For the simulations we assumed that on average subjects intended to hit the disk at its position at the average time that the stylus reached the distance at which such disks are hit (3rd row of Table 1). Moreover they plan to hit the disk through the center of the goal, so that they plan to hit it at the position along its edge that would get it to the centre of the goal considering the average motion of the stylus at that time (1st and 2 nd rows). Although presenting both $\sigma_{\text {time }}$ and $\sigma_{\text {disk }}$ is redundant, because the disk always starts at the same distance and moves at the same speed, both are shown in Table 1 to facilitate the comparison with other values. The standard deviation in the time taken to hit the disk (rather than to reach the appropriate distance) is $44 \mathrm{~ms}$ when hitting further and $46 \mathrm{~ms}$ when hitting back.

Figure 5 shows the most critical parameters for success: where the disk is at the moment that it is hit, and the direction in which it moves after being hit. The shaded areas show the combinations of these parameters for which the disk will pass through the goal. It is evident from the scatter of the data points relative to the shaded area that hitting the disk in the correct direction is more of a problem than hitting it when it is at an appropriate position. This is not surprising because small errors in where one hits the disk's surface can make it move in a very different direction. Our simulations (the smaller panels on the right) reproduce much of the observed pattern in performance, although the data for hitting back contain a substantial number of trials in which subjects appear to have tried to hit later (more negative values of the lateral position) and further from the side (so that the disk moves in a direction that is closer to $90^{\circ}$ ). We think that the pattern in the simulated data is similar enough to the measured data to conclude that our simulation considers the main factors that affect performance.

For further validation of the assumptions that we made when designing our simulations we compared measured data with values predicted by the simulations for several parameters that were not considered for the simulations. Subjects missed the disk altogether 13 times when hitting further (1.6\% of trials) and 11 times when hitting back $(1.4 \%)$. The simulation predicts values of about $5 \%$ and less than $1 \%$. The average standard deviation in the position of the disk at the moment that it was actually hit was $0.87 \mathrm{~cm}$ when hitting further and $0.92 \mathrm{~cm}$ when hitting 
Fig. 4 Average paths (a) and velocity profiles (c) in the four conditions (b). The shaded areas show standard errors across the nine subjects. The disks are shown at their average positions when hit. Since the paths for mirror-symmetric conditions are approximately mirror-symmetric (a), and their velocities are similar $(\mathbf{c})$, we averaged across mirror symmetric conditions after flipping them laterally (d). Red indicates disks that are hit further and blue ones that are hit back

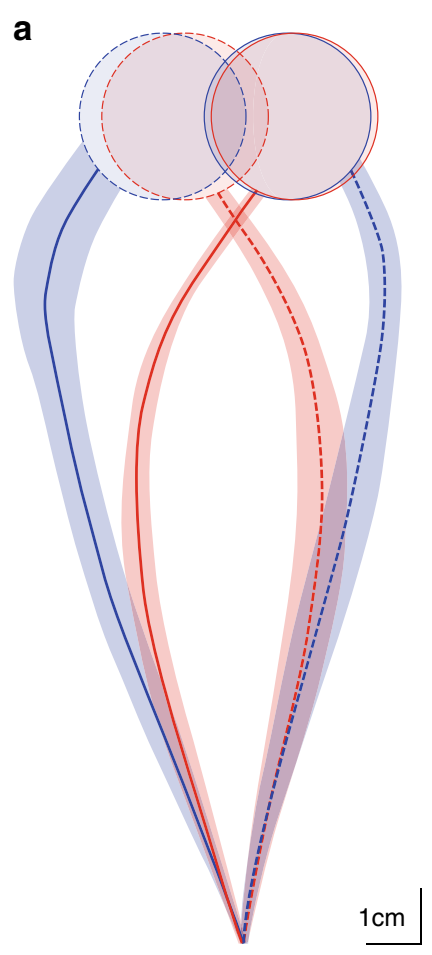

b

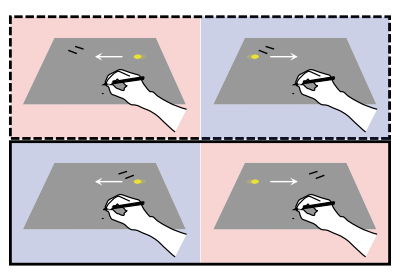

C

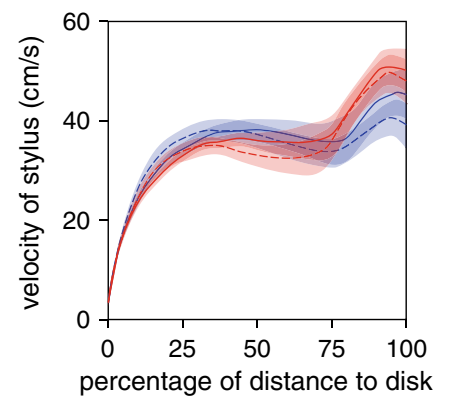

d

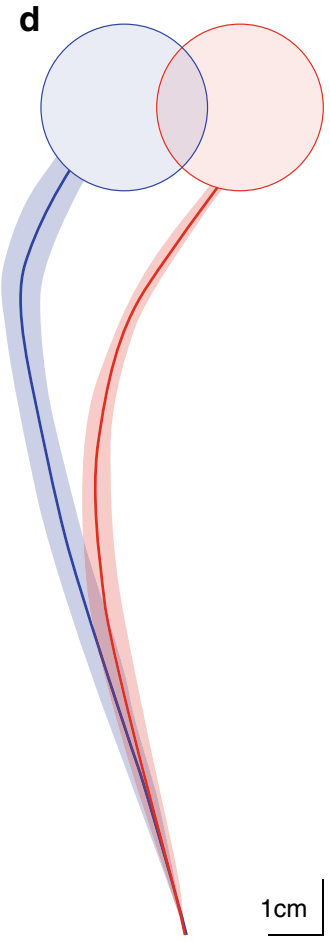

Table 2 Estimates of variability derived from Eqs. 1-3

\begin{tabular}{ll}
\hline$\sigma_{\mathrm{s}}(\mathrm{cm})$ & 0.44 \\
$\sigma_{\text {plan }}(\mathrm{cm})$ & 0.79 \\
$\sigma_{\mathrm{t}}(\mathrm{ms})$ & 22 \\
\hline
\end{tabular}

back (the simulation predicts 0.96 and $0.86 \mathrm{~cm}$ ). The standard deviation in the hit position along the edge of the disk is $21^{\circ}(5.5 \mathrm{~mm})$ when hitting further and $17^{\circ}(4.5 \mathrm{~mm})$ when hitting back (the simulation predicts $24^{\circ}$ and $16^{\circ} ; 6.3$ and $4.2 \mathrm{~mm}$ ). The values from the simulations are quite close to the measured values, although subjects tend to perform slightly better than the simulation predicts when hitting further and slightly worse when hitting back. We also examined the correlations between three of the abovementioned parameters, both in the data and in our simulations (Fig. 6). The pattern of correlations in the data (solid symbols) is very similar to that in the simulations (open symbols), so a combination of the geometry of the task and the three independent sources of variability (with the values given in Table 2) is enough to account for this aspect of performance as well.

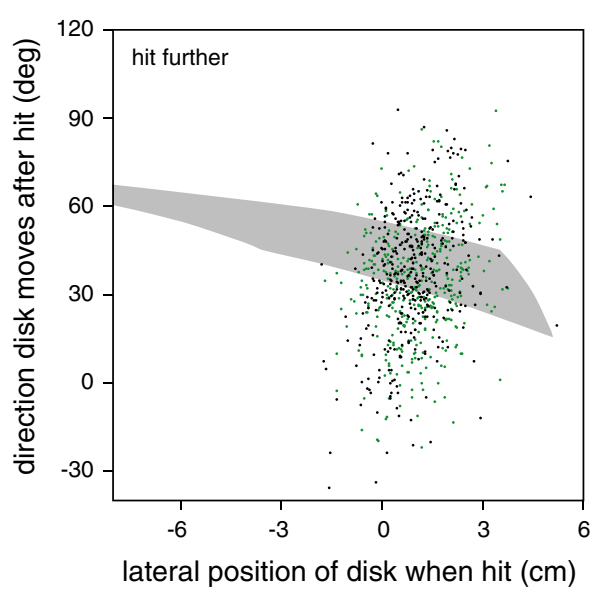

Fig. 5 Geometrical limitations. The shaded area shows the range of values for which the disk will pass through the goal. The dots in the main panels each represent a single trial. Black dots show data for the conditions with the goal on the right and green dots show (flipped)
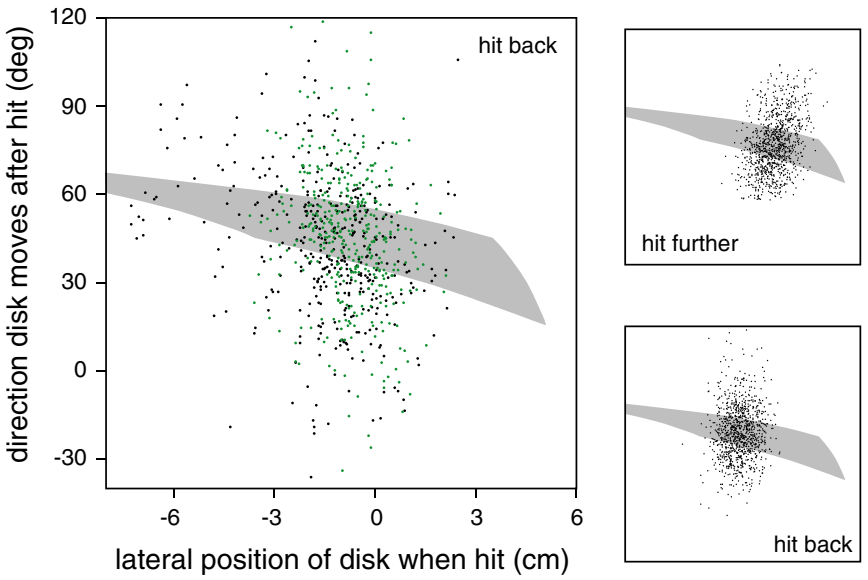

data for the conditions with the goal on the left. There are a few points outside the visible range. Each of the smaller panels on the right shows the outcome of 1000 simulations (for details see Methods) 


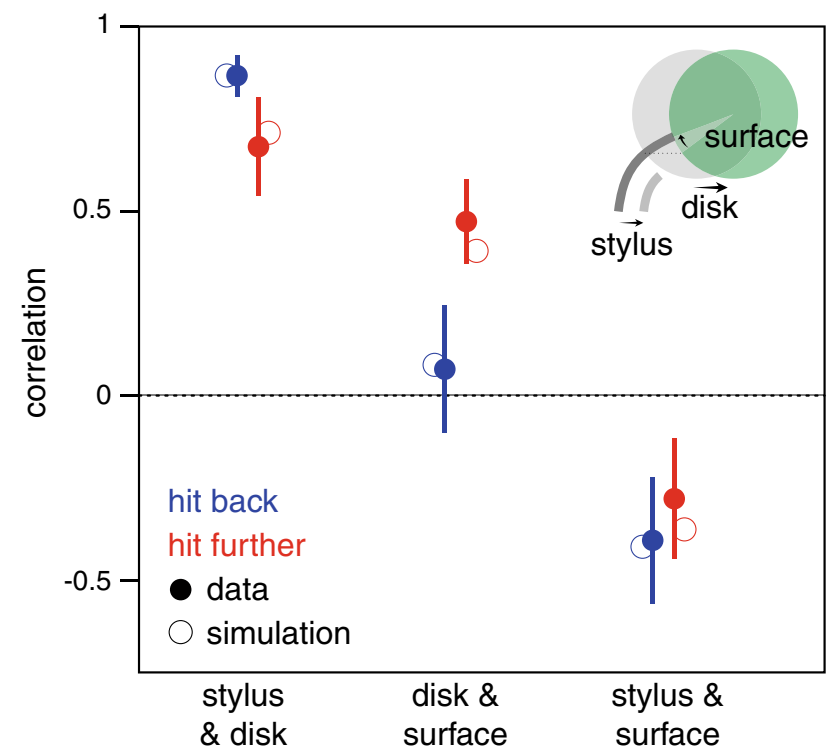

Fig. 6 Correlations between the position of the disk when it is hit (disk), the position along its edge at which it is hit (surface), and the lateral position of the stylus as it crosses the distance at which it is expected to hit the disk (stylus). Error bars are 95\% confidence intervals across the nine subjects (after averaging across goal positions). The open symbols are predictions from simulations based on the average measured movement parameters and three independent sources of variability (see Table 2)

Having established from Fig. 5 that the main problem for the subject is to hit the disk in the correct direction, we can examine why this is so. The direction in which the disk moves after impact depends both on where on the disk's edge the stylus hits it and on the speed of the impact. The latter is the component of the relative motion between

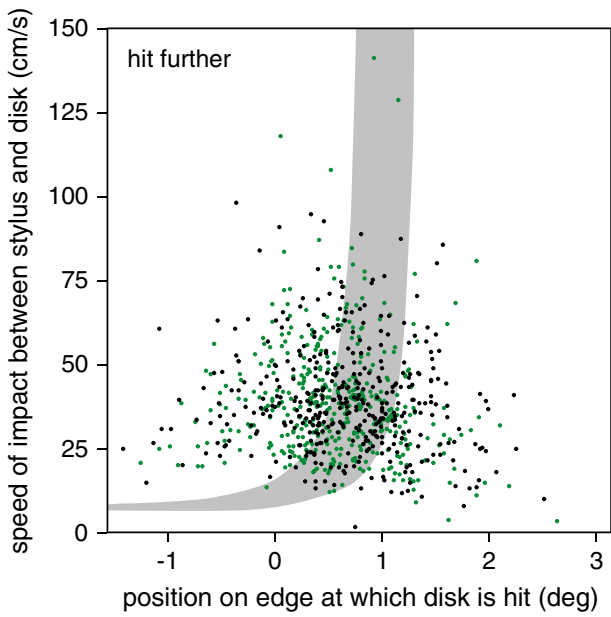

Fig. 7 Precision and speed of hit. The direction in which the disk moves after impact depends on where along its edge the stylus makes contact with it and the speed of the impact. The latter is the component of the relative motion between stylus and disk that is orthogonal to the edge at the point of contact. The position along the stylus and disk that is orthogonal to the disk's edge at the point of contact. Figure 7 illustrates that hitting the correct position on the disk's edge is generally more critical than ensuring that one is moving at the correct speed or in the correct direction when one hits it. The very low impact speed on occasional trials arises when the stylus skims the disk (so that the velocity orthogonal to the surface is very small). The speed of impact is higher when hitting back because the disk and stylus are moving in opposite directions. It is evident from the scatter of the data points relative to the shaded area that hitting the right part of the disk is more difficult than hitting it with an appropriate speed. The pattern of data points is also consistent with this (assuming that the plan is for the stylus to approach the disk's edge more or less perpendicularly) because hitting the wrong part of the disk generally reduces the angle at which one does so, so the speed of impact is lower. Thus, larger errors in the hit position of the disk's edge should be associated with a lower impact speed, as indeed they are.

We assumed for our simulations that the variability in the speed and direction of the stylus' movement when it hit the disk would not influence performance very much. This was confirmed by including such variability in new simulations. These simulations were identical to the initial ones but we selected a random direction and speed for each simulated trial from normal distributions with the average values and standard deviations given in Table 1 (instead of always using the average value). Doing so made very little difference to any of the reported measures. This implies that subjects have some freedom to vary the path and speed across trials, which they may use to adjust ongoing movements to ensure that the stylus reaches the intended contact

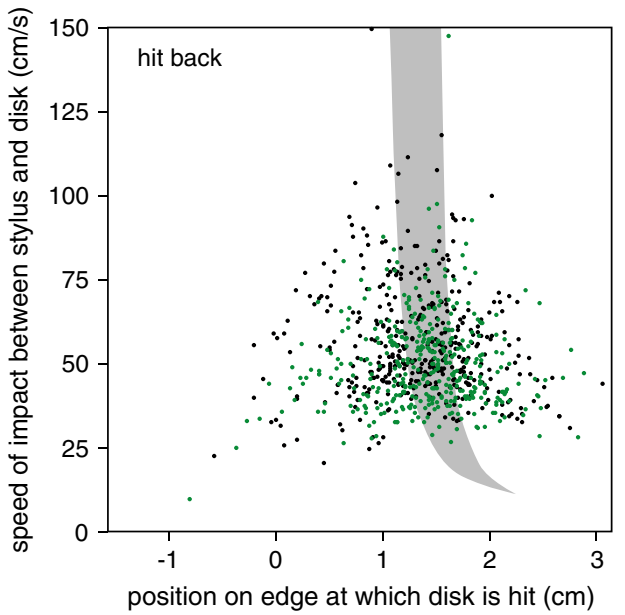

disk's edge is measured from the point that passes closest to the starting point, with positive values representing the direction that is appropriate for making the disk move toward the goal. For further details see Fig. 5 
point at the intended moment. Part of the measured variability in the stylus' motion may result from such adjustments, although part of it is undoubtedly the result of failing to perform the movement exactly as planned, and part may be intentional, for instance because the optimal direction and speed depend on the disk's position when it is hit.

To try to get some idea of how the variability develops during the movement we examined two additional parameters. An obvious parameter to examine is the reaction time. The standard deviation in the reaction time was $59 \mathrm{~ms}$ when hitting further and $55 \mathrm{~ms}$ when hitting back (which is larger than that in the time to reach the disk's distance). Subjects compensated for starting later by moving faster (correlations of 0.22 and 0.23 between reaction time and the stylus' speed when it reaches the disk, for hitting back and further, respectively). However, when they started later they also hit the disk further along its path (correlations of -0.37 and 0.30 between reaction time and the stylus' lateral position when it reaches the disk, for hitting back and further). We also examined the lateral variability in the stylus' position when it was half way to the disk, and found that it was $0.91 \mathrm{~cm}$ when hitting further and $1.13 \mathrm{~cm}$ when hitting back (which is larger than the variability at the distance of the disk).

\section{Eye movements}

For two subjects the recordings failed: the calculated gaze directions were clearly meaningless. Perhaps they knocked the headset against the setup, shifting the cameras relative to the eyes. For one subject the eye movement calibration failed. On 120 of the remaining trials $(10 \%)$ we missed gaze for some time either because the subject blinked or because the head moved outside the range within which its position and orientation could be measured. On 22 trials the stylus missed the disk. Figure 8 shows the average velocity of eye and head movements in the remaining 1058 trials. In our setup a translation of $0.8 \mathrm{~cm} / \mathrm{s}$ is about equivalent to a rotation of $1 \% \mathrm{~s}$ in terms of gaze shift. The figure shows that most, but not all, of the change in gaze is due to eye movements.

For determining what the subject was looking at, we not only removed gaze traces in which parts of the eye or head movement recordings were missing and ones in which the subject missed the disk, but also ones in which the subject was ever looking somewhere other than toward one of the places that we consider relevant to the task (i.e., not at the stylus, disk, goal, starting point, or point of interception). Doing so left us with 547 trials. Figure 9 shows an example of a successful trial (in terms both of recording gaze and hitting the disk). In this case the subject made a single saccade from the starting point to the disk, and then pursued the disk until the stylus hit it.

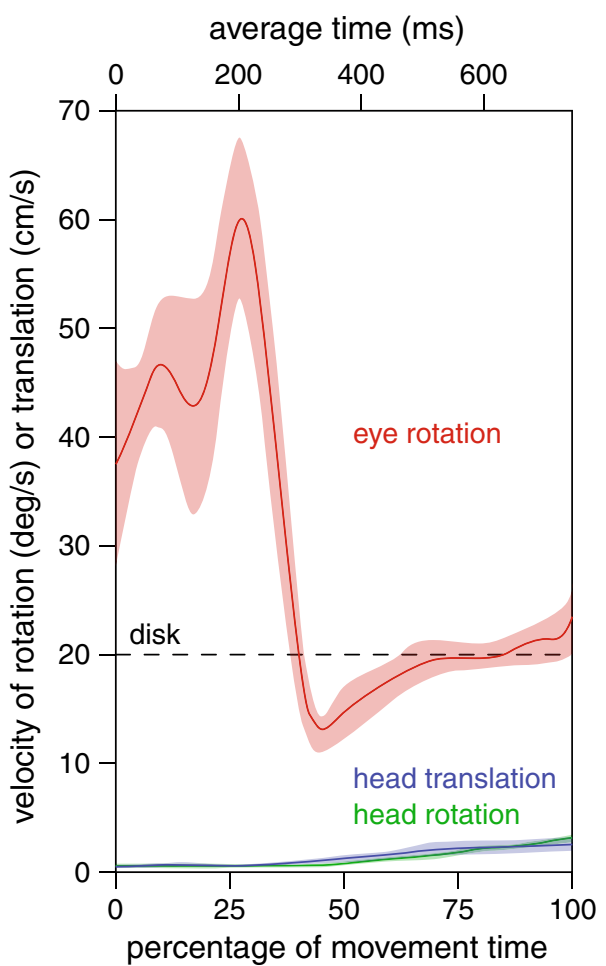

Fig. 8 Average velocity at which the eyes (red) and head (green) are rotating, and at which the head is translating (blue), at each moment during the interceptive movement (with the standard errors across the six subjects). Before averaging, the motion of the eyes and head were re-sampled as a function of the movement time (of the disk rather than of the stylus, with $100 \%$ representing the moment the disk is hit by the stylus). For each subject, the horizontal and vertical rotations, and the translations in all three directions, were averaged separately for each condition, converted into a single magnitude of average rotation or translation, and then the absolute values were averaged across conditions. The axis at the top shows the average time from the moment the disk appears. The dashed line shows the disk's velocity (in $\mathrm{cm} / \mathrm{s}$ )

This pattern of eye movements was found in about half of the trials. Figure 10 shows the relative frequencies of the six sequences of eye movements that we found in the 547 trials for which we were sure about where subjects were looking. Since gaze had to be directed toward the starting point to start a trial, most trials started with the eyes directed toward the starting point, followed by a saccade upwards, toward the region where the disk and goals appeared (as in Fig. 9). However, on some trials the eyes started moving before the disk appeared, so that the trial started with an upward saccade. These two possibilities are pooled in Fig. 10.

The results shown in Fig. 10 are for the half of the trials for which we are certain about what the subjects were looking at. If we assume that subjects did not change where they were looking when data was missing for short periods of time (due to blinks) and if we are less strict with our criteria (e.g., considering pursuit parallel to-but slightly 
Fig. 9 Motion of the disk, the stylus and gaze during a single trial. Traces are shown from when the disk appeared until the stylus hit it. The paths on the tablet are shown on the left. The timing is shown on the right, with separate traces for sagittal and lateral position as a function of time. The traces are color coded, with gray for the disk, blue for the stylus, pink for fixation, red for pursuit and green for the (single) saccade

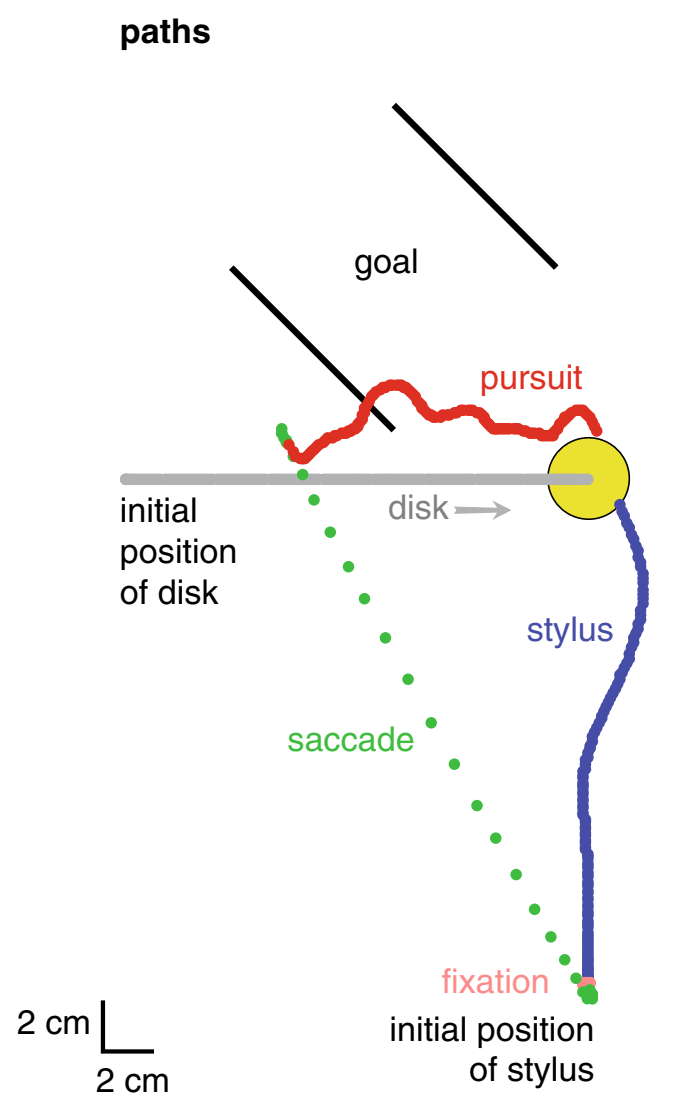

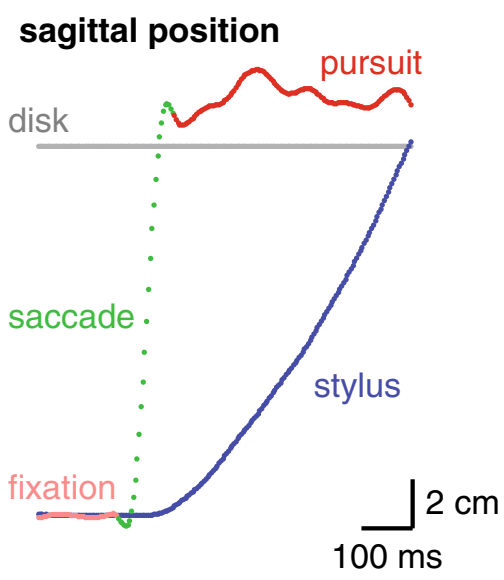

lateral position

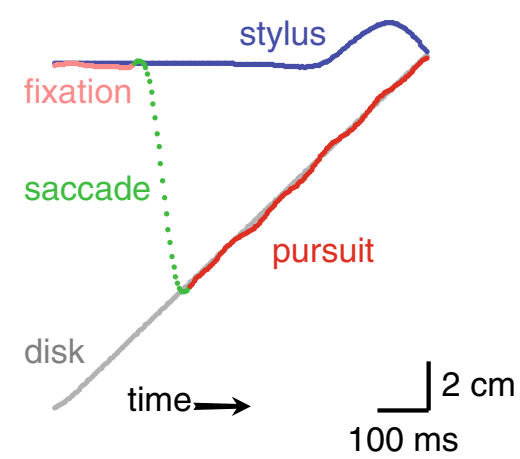

more than $5 \mathrm{~cm}$ from-the disk as pursuing the disk) we could interpret 1027 trials. Considering trials in which we are less certain shows approximately the same pattern, but the simplest sequence (making a saccade to the disk and then pursuing it until contact) is slightly more frequent and there are several trials with no identifiable pursuit. In the few trials in which even less strict criteria left subjects looking in an unidentified direction, subjects may have made a saccade to the side at which they expected the disk to appear when it actually appeared at the other side, they may have underestimated a required saccade amplitude, or they may have briefly looked elsewhere for reasons that are not related to the task.

Figure 11 gives an impression of how soon gaze left the starting point and how much time was spent pursuing the disk. Here again all 1058 trials without missing data are included. When performing everyday tasks our eyes are usually directed at the object or objects that are relevant for what we are doing at that moment (Hayhoe et al. 2003; Johansson et al. 2001; Land et al. 1999; Land and Hayhoe 2001; Triesch et al. 2003), or toward positions at which critical information is expected to become available (e.g., information about how a ball bounces; Land and Furneaux 1997; Land and McLeod 2000). We could therefore tentatively conclude from Fig. 11 that the disk is particularly relevant for this task. However, the goal is obviously also relevant, and on many trials it was not fixated at all, or only after having hit the disk. Subjects probably fixated the disk most of the time because it is critical to hit exactly the right place on its edge. Moreover, the fact that the disk was moving may have made it particularly important to keep ones eyes on it. The position of the static goal may have been determined accurately enough in peripheral vision or during the quick glimpse that subjects took on many trials. We do see quite a few saccades toward the goal from about $80 \mathrm{~ms}$ before the stylus hit the disk (i.e., from about $90 \%$ of the movement time in Fig. 11), which is about when visual information is no longer useful for guiding the hand to the best position on the disk. Presumably they are important for obtaining feedback about ones own performance.

\section{Discussion}

The present study examines variability in an interception task. We found that a simple simulation with three sources of variability can reproduce many characteristics of performance near when the stylus hits the disk, which is the critical moment for success in the task. This suggests that these are the main sources of variability. It is therefore interesting to examine which sources of variability we did 


\section{frequency of occurrence of various patterns of eye movements}

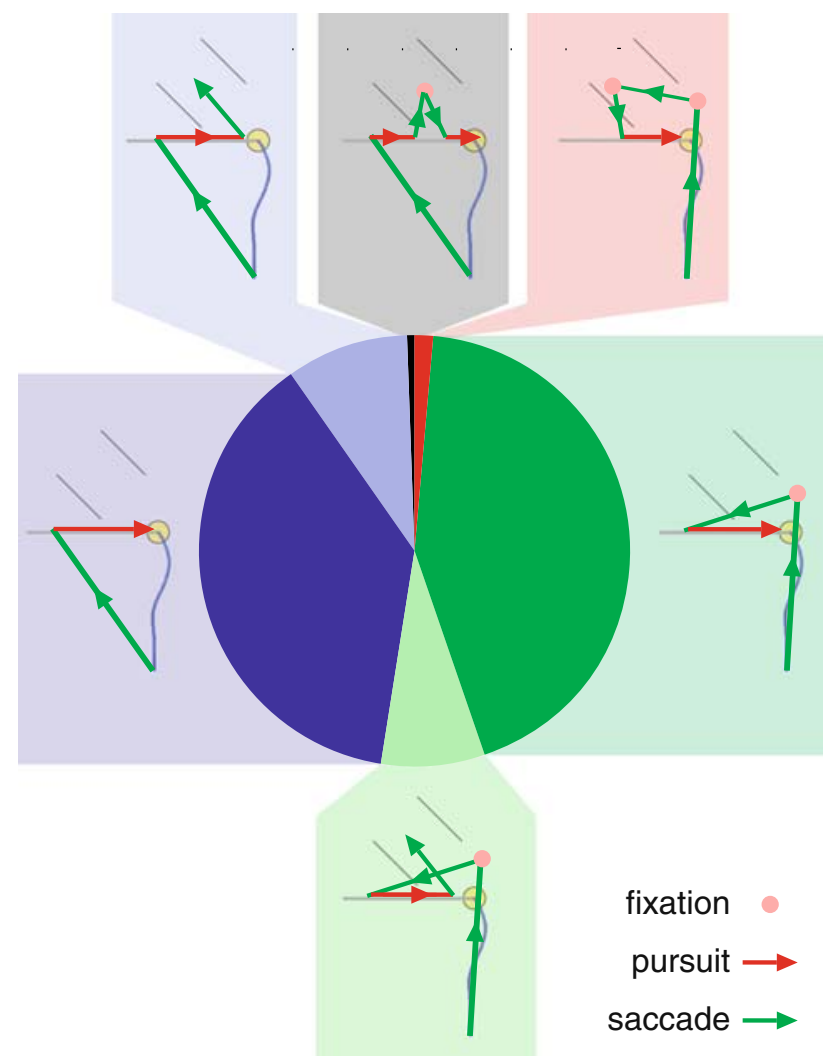

Fig. 10 The six sequences of eye movements that we found and their relative frequencies. The sequences are considered from the saccade away from the starting point until the stylus hits the disk. The schematic illustrations are for a disk moving to the right that is hit back toward a goal on the left, but all four conditions are considered. The blue sections represent trials in which subjects made a saccade to the disk and pursued it until they hit it (dark blue) or until just before they hit it, at which time they made a saccade to the goal (light blue). The green sections represent trials in which subjects made a saccade toward the goal before pursuing the disk, with the brightness indicating the same distinction. The red section represents trials in which subjects made an additional saccade toward the goal before pursuing the disk. The black section represents trials in which pursuit of the disk was interrupted by a saccade toward the goal

not consider and which characteristics of the data are not reproduced.

The most obvious variability that we ignored is the variability between subjects. For each measure that we examined, the standard deviation for the person with the largest standard deviation was about twice that for the person with the smallest. The number of 'goals' scored varied between 51 and 93 . Thus, there are certainly differences between subjects. Since subjects differ in their perceptual and motor precision (as well as in their eagerness to score goals) these differences are not particularly surprising. They do of course imply that the spatial and temporal errors that we report in Table 2 are only indications of the precision that can be achieved, because the true values differ between subjects.

We assumed that the three key sources of variability are independent of whether one is hitting further or back. The fact that our simulations reproduce many of the observed differences between hitting further and back (see Figs. 5, 6, 7) shows that these differences are a consequence of the different geometry of the two conditions rather than of differences in variability. However, our subjects' performance was often slightly better than the simulation when hitting further and worse than the simulation when hitting back. This may be because the disk had to be hit slightly more from the side in order to hit it back. We have previously shown that following a more curved path leads to larger endpoint variability (Brenner and Smeets 2007), possibly due to more variability in muscle activation (van Beers et al. 2004; Harris and Wolpert 1998). However, the path is not much more curved when hitting back (Fig. 4a). There may also be more spatial variability when hitting back because the retinal eccentricity of the hand is larger as it approaches the disk for hitting it back, which could lead to more spatial variability if visual information about the moving hand is used to guide the stylus to the disk.

Variability in the plan

Our analysis is based on what happens near the time the stylus hits the disk. We separated random spatial and temporal variability that is detrimental to performance from 'planned' variability that is not (because the way the stylus moves is appropriate for the time and place at which the disk is to be hit). Many factors undoubtedly contribute to the latter, including adjustments made during the movement. One factor that is certainly important on early trials is that subjects must learn how the disk will respond to the impact with the stylus. Fluctuations in the estimate of how the disk responds to being hit will result in variability in the movements. Subjects may initially even intentionally modify the movement between trials to discover how they can best hit the disk, because without doing so they may not become aware of certain strategies being more suitable than others. We reduced the influence of this factor by giving the subjects practice trials and later removing the first 20 trials because performance had not yet stabilized.

Performance in our task was largely determined by whether the stylus hit the right point on the disk's edge. Thus, subjects may primarily be trying to hit the right part of the disk, and the variability in the disk's position when it is hit may mainly emerge from adjusting the movement of the stylus to achieve this goal. The fact that the eyes pursue the disk most of the time supports this view. If so, then what we called variability in the plan may largely be due to adjustments that are made during the movement. Such 


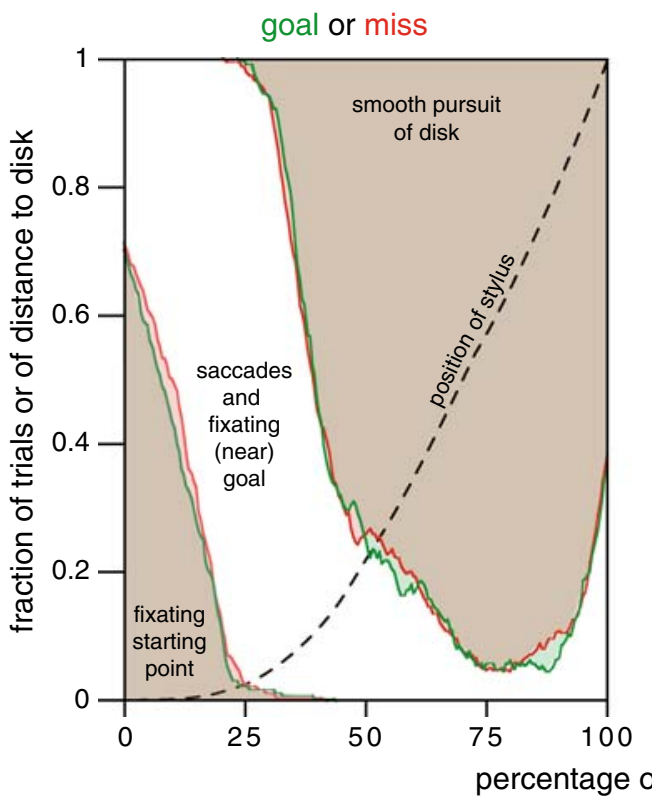

Fig. 11 Timing of the first saccade onset and pursuit of the disk. The shaded parts of the figures show the time spent fixating the starting point before the first saccade and the time spent pursuing the disk. The remaining time is spent making saccades (including catch-up saccades) and fixating structures of interest (mainly the goal). Movement time refers to movement of the disk rather than of the stylus (with $100 \%$ representing the moment the disk is hit by the

adjustments could be necessary to compensate for the variability in the reaction time (which could partly arise from the disk appearing at the other side than expected or the subject still trying to precisely position the stylus at the starting point when the disk appeared). However, subjects may intentionally start moving later on some trials, in order to hit the disk further along its path, or in order to be moving faster when they hit it. The finding that the lateral variability was larger half way to the disk than that at the distance at which the disk was hit (although the stylus always started at the same position) suggests that subjects may have planned to follow differently curved paths on different trials, but the variability may also be larger half way to the disk because the task constrains the acceptable endpoints much more severely than it does the path toward these endpoints, so the path is adjusted to ensure that the disk is hit when it is at an appropriate position.

\section{Vision as a limiting factor}

It is well-known that performance deteriorates if vision is severely limited (e.g., by occluding vision during certain intervals or by not allowing subjects to direct their gaze toward the target) or if vision provides incorrect information (e.g., by having subjects look through prisms). It has also been argued that vision limits performance in sports in performance on first, second, third and last 50 trials

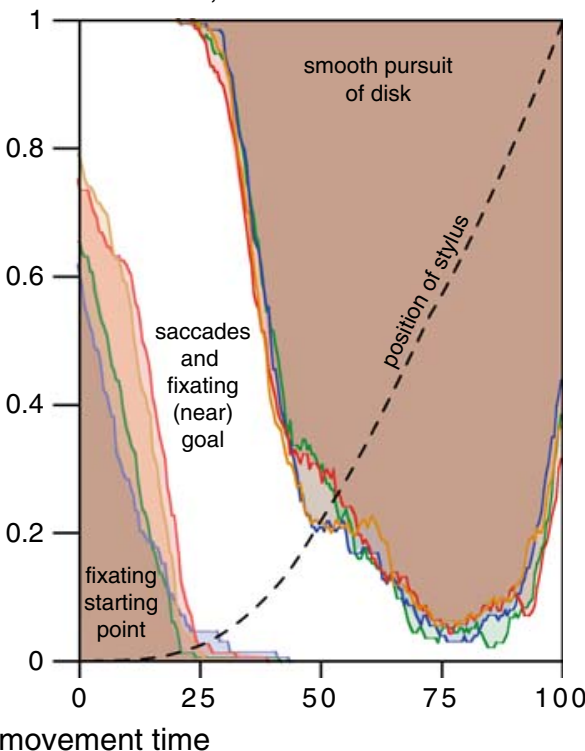

stylus). The dashed line shows the fraction of the distance to the disk that the stylus has covered as a function of the movement time. We see no difference between successful and unsuccessful trials (left panel). During the course of the experiment subjects make their first saccade earlier, but the time spent pursuing the disk does not change. Just before the stylus hits the disk there is an increase in the number of saccades toward the goal. Average data of six subjects

which balls move in depth (visual resolution is particularly poor in depth; Brenner and Smeets 2000) and are intentionally thrown in a way that makes visual judgments as difficult as possible (reviewed in Regan 1997). However it is not clear to what extent vision limits performance in interception under conditions that are more favorable for visual judgments. In our experiment the target was always clearly visible as it moved laterally at a modest constant velocity, which should be favorable conditions for vision.

Our estimate of the lateral spatial precision is $4.4 \mathrm{~mm}$ (Table 2). How much of this is likely to be of visual origin? People are known to have a visual resolution of about 1 minute of arc, and to detect even smaller differences under certain conditions, but such precision is only achieved when retinal resolution is the limiting factor. We know that fast movements are not guided on the basis of relative retinal positions (Brenner and Smeets 2003, 2006). In order to judge the position of the disk (or the stylus), one must consider the orientation of the eyes and head. Van Beers et al. (1998) estimated that the standard deviation for visually localizing static targets on a table-top is about $0.4^{\circ}$, which would correspond in our experiment to about $3 \mathrm{~mm}$. We previously found standard deviations between 3 and $5 \mathrm{~mm}$ (depending on the direction in which the target was to be hit) for hitting static targets under similar conditions to those used here (Brenner and Smeets 2007). 
Trommershauser et al. (2003) found standard deviations of about $4 \mathrm{~mm}$ after some practice (second session) under quite different conditions, but with a similar target distance, reaction time and movement time. If we combine the above-mentioned estimate of $3 \mathrm{~mm}$ for visually localising the target with an estimate of $4 \mathrm{~mm}$ for hitting static targets we obtain an estimate of $2.6 \mathrm{~mm}$ for the control of the moving hand (stylus).

In our experiment the disk was moving, so a visual estimate of its position must be combined with an estimate of its velocity to judge its position at some time in the future. De Bruyn and Orban (1988) estimated that for the velocity of our disk (about $24 \%$ s) one needs a $5 \%$ change in velocity to correctly identify which of two targets is faster on $84 \%$ of the trials. This corresponds with a standard deviation in the velocity estimate of $1 \mathrm{~cm} / \mathrm{s}$. How much the endpoint of the movement can be expected to vary as a result of misjudging the velocity depends on the time across which one uses the velocity to predict the position at which the disk will be hit. For the minimal delay for transforming visual information into an action (about $110 \mathrm{~ms}$ for a response to a change in position; Brenner and Smeets 1997), the additional variability would only be about $1.1 \mathrm{~mm}$. Combining this with $3 \mathrm{~mm}$ for visually localising the target (from the previous paragraph) would only increase the visual contribution to the spatial variability to $3.2 \mathrm{~mm}$ (assuming that errors in judging location and velocity are independent), and combining that with the estimate of $2.6 \mathrm{~mm}$ for the control of the moving hand would predict a lateral spatial error of $4.1 \mathrm{~mm}$. However, the above-mentioned $110 \mathrm{~ms}$ delay is for the very first response. Considering that not all responses are so fast, and that it also takes time for a response to have an effect, the appropriate value may be higher. Predicting the point of interception $200 \mathrm{~ms}$ in advance would fully account for the lateral spatial error $\left(\sigma_{\mathrm{s}}\right)$ of $4.4 \mathrm{~mm}$. Predicting the endpoint at the beginning of the movement would increase the visual contribution to $5.6 \mathrm{~mm}$ and give a lateral spatial error of $6.2 \mathrm{~mm}$. Although these values are all approximations, they suggest that the movement is adjusted until the last possible moment and that vision contributes substantially to the lateral spatial error. The role of vision is especially evident if one considers that our estimated $2.6 \mathrm{~mm}$ for variability in the control of the moving hand is likely to include a contribution from vision of the hand.

Interpreting the temporal variability is more complicated, because our estimate of $22 \mathrm{~ms}$ (Table 2) is not only a combination of the precision in judgments about the disk and the hand (or stylus), but also combines true temporal variability $\left(\sigma_{\mathrm{t} \text {-true }}\right)$ with variability in timing that arises from spatial errors along the stylus' path $\left(\sigma_{\text {path }}\right)$. The two sources of variability cannot easily be separated, but we can examine the possibilities by considering which splits would be plausible. If the two sources of variability are independent, then

$\sigma_{\mathrm{t}}^{2}=\sigma_{\mathrm{t}-\text { true }}^{2}+\frac{\sigma_{\text {path }}^{2}}{V_{\text {stylus }}^{2}}$

where $V_{\text {stylus }}$ is the velocity of the stylus. Filling in the calculated value of $22 \mathrm{~ms}$ (from Table 2) for $\sigma_{\mathrm{t}}$ and the average value of $47.3 \mathrm{~cm} / \mathrm{s}$ for $V_{\text {stylus }}$ (from Table 1 ), and plotting the resulting possible values of $\sigma_{\text {t-true }}$ and $\sigma_{\text {path }}$, we get the thick curve that is shown in Fig. 12.

Although all points along the thick curve in Fig. 12 are consistent with our data, some are less likely than others. We can use the same reasoning as we used to analyse the various contributions to the lateral spatial variability $\left(\sigma_{\mathrm{s}}\right)$ to estimate reasonable values of $\sigma_{\text {path }}$. The visual contribution to the variability in localizing the target at a given moment is larger in the sagittal direction because of the angle between the surface and the line of sight, but errors in judging the disk's speed are irrelevant and the influence of errors in judging its direction of motion are probably negligible. We do not know how the felt position of the moving hand is affected. Thus, we can only really be confident that the spatial variability is larger than the prediction for a visual resolution of $0.4^{\circ}$ and viewing the surface at an angle of $45^{\circ}$ (i.e., than the $4.2 \mathrm{~mm}$ indicated by the shading in Fig. 12).

In a similar study to ours, but in which subjects moved much faster because they only had to worry about timing the interception, there were conditions in which the variability in the timing was only about $12 \mathrm{~ms}$ (Tresilian and

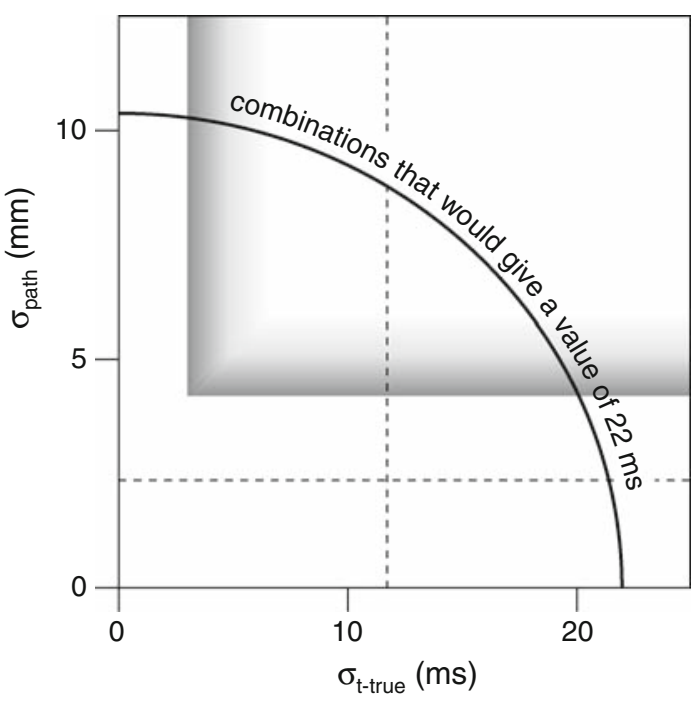

Fig. 12 Distinguishing temporal from spatial contributions to $\sigma_{\mathrm{t}}$. The thick curve shows all possible combinations based on Eq. 4. The shading indicates the minimal values of $\sigma_{\text {path }}$ and $\sigma_{\text {t-true }}$ that we consider reasonable. The dashed lines show the spatial and temporal steps between successive positions of the disk 
Plooy 2006). A standard deviation in timing of only $6 \mathrm{~ms}$ was found for hitting a dropping ball with a bat (McLeod and Jenkins 1991; McLeod et al. 1985). That faster movements are timed more accurately (also see Schmidt 1969; Wallace et al. 1990) suggests that much of the timing error is of spatial origin, because the influence of misjudging the distance to the target is smaller if one moves faster. Most direct tests of the temporal resolution of vision, such as judgments of whether or not two flashes occurred simultaneously (Virsu et al. 2008) and temporal order judgments (Jaskowski and Verleger 2000), find values that correspond with standard deviations of tens of ms, but Westheimer and McKee (1977) found conditions for which the temporal resolution corresponds with a standard deviation of only about $3 \mathrm{~ms}$. Under those conditions performance is probably based on a motion signal rather than on separate judgments of timing. Presumably interception also relies on temporal information that is not accessible for simultaneity and temporal order judgments. It is worth noting that performance was close to the resolution of our setup, with a new image being shown every $12 \mathrm{~ms}$ (dashed lines in Fig. 12). Although our analysis is based on interpolated values we cannot be sure that the resolution of the display did not limit performance as well.

From all these calculations it would appear that a substantial part of our subjects' spatial and temporal variability is of visual origin. Moreover the prediction of the disk's position at the expected moment of the hit must have been updated throughout the movement, because considering the resolution of visual velocity judgments, and that the influence of misjudging the velocity on the prediction is proportional to the duration for which motion is extrapolated, misjudging the disk's velocity would otherwise have overshadowed all other effects (unless interception uses velocity information that was not accessible for the velocity discrimination judgments of de Bruyn and Orban 1988). Thus in many sports situations visual resolution is likely to limit performance. However, people probably generally control their movements in a way that minimizes the total error, so they modify their strategy to reduce the factor that introduces the largest errors, even if this means that other errors increase. Thus it is possible that completely different factors are critical in different kinds of interception tasks.

\section{The strategy}

Subjects systematically hit the disk after it had passed the midline, even when hitting it back, suggesting that they would have preferred to move more slowly, because hitting the disk back later means one has to be more accurate (see Fig. 5; hitting back later corresponds to negative values, where the vertical extent of the shaded area is smaller). The optimal speed for intercepting a target is one that finds the right balance (Brouwer et al. 2000) between moving slowly to improve the spatial resolution (Fitts and Peterson 1964) and moving fast to improve the temporal resolution (Schmidt 1969). The fact that the disk had to pass through the goal probably forced our subjects to move faster than would be optimal for hitting a chosen spot on the target's edge. The reaction times were quite short, so subjects could not start moving much earlier in order to be able to move more slowly. Thus we may have found better performance if the disks had started further from the midline. It is evident that the $22 \mathrm{~ms}$ and $4.4 \mathrm{~mm}$ from Table 2 only provide an indication of the kind of precision that people can achieve in interception tasks. Undoubtedly there will be conditions in which (certain) people will perform significantly better or worse. We especially expect worse performance under conditions in which visual spatial judgements are less precise, such as for targets moving in depth (but see suggestions to the contrary for professional batters in Regan 1997).

When thinking about how movements are planned we usually think of strategic decisions. Harris and Wolpert (1998) suggested that a strategy is chosen that optimizes task performance in the face of signal dependent noise in regulating muscle activity. Churchland et al. (2006) raised doubts about whether limitations in the resolution with which muscle activity can be controlled are the primary reason for repeated movements being variable. They showed that much of the variability in performance is already visible in the dorsal premotor and primary motor cortex. Our results suggest that much of the variability arises when judging the position and motion of the disk (and hand). Presumably variability is introduced at all stages of neural processing leading from sensory information to muscle activation. Part of the strategy in converting sensory information and past experience into a movement plan is presumably aimed at reducing the influence of such variability at the critical moment. In our task, where performance relied on hitting the correct part of the disk's edge, continuously updating visual judgments of the disk's position and velocity was probably crucial for anticipating the disk's position at the moment of impact, as was pursuing the disk with the eyes to maximize the spatial resolution of such judgments.

Open Access This article is distributed under the terms of the Creative Commons Attribution Noncommercial License which permits any noncommercial use, distribution, and reproduction in any medium, provided the original author(s) and source are credited.

\section{References}

Biegstraaten M, Smeets JBJ, Brenner E (2006) The relation between force and movement when grasping an object with a precision grip. Exp Brain Res 171:347-357 
Brenner E, Smeets JBJ (1995) Moving one's finger to a visually specified position: target orientation influences the finger's path. Exp Brain Res 105:318-320

Brenner E, Smeets JBJ (1997) Fast responses of the human hand to changes in target position. J Mot Behav 29:297-310

Brenner E, Smeets JBJ (2000) Comparing extra-retinal information about distance and direction. Vision Res 40:1649-1651

Brenner E, Smeets JBJ (2003) Fast corrections of movements with a computer mouse. Spat Vis 16:365-376

Brenner E, Smeets JBJ (2006) Two eyes in action. Exp Brain Res 170:302-311

Brenner E, Smeets JBJ (2007) Flexibility in intercepting moving objects. J Vision 7:14.1-17.0

Brouwer AM, Brenner E, Smeets JBJ (2000) Hitting moving objects. The dependency of hand velocity on the speed of the target. Exp Brain Res 133:242-248

Churchland MM, Afshar A, Shenoy KV (2006) A central source of movement variability. Neuron 52:1085-1096

de Bruyn B, Orban GA (1988) Human velocity and direction discrimination measured with random dot patterns. Vision Res 28:1323-1335

de Lussanet MH, Smeets JBJ, Brenner E (2001) The effect of expectations on hitting moving targets: influence of the preceding target's speed. Exp Brain Res 137:246-248

Fernandez L, Bootsma RJ (2004) Effects of biomechanical and task constraints on the organization of movement in precision aiming. Exp Brain Res 159:458-466

Fitts PM, Peterson JR (1964) Information capacity of discrete motor responses. J Exp Psychol 67:103-112

Goodale MA, Pélisson D, Prablanc C (1986) Large adjustments in visually guided reaching do not depend on vision of the hand or perception of target displacement. Nature 320:748-750

Harris CM, Wolpert DM (1998) Signal-dependent noise determines motor planning. Nature 394:780-784

Hayhoe MM, Shrivastava A, Mruczek R, Pelz JB (2003) Visual memory and motor planning in a natural task. J Vision 3:49-63

Jaśkowski P, Verleger R (2000) Attentional bias toward low-intensity stimuli: an explanation for the intensity dissociation between reaction time and temporal order judgment? Conscious Cogn 9:435-456

Johansson RS, Westling G, Backstrom A, Flanagan JR (2001) Eye-hand coordination in object manipulation. J Neurosci 21:6917-6932

Land MF, Furneaux S (1997) The knowledge base of the oculomotor system. Philos Trans R Soc Lond B Biol Sci 352:1231-1239
Land MF, Hayhoe M (2001) In what ways do eye movements contribute to everyday activities? Vision Res 41:3559-3565

Land MF, McLeod P (2000) From eye movements to actions: how batsmen hit the ball. Nat Neurosci 3:1340-1345

Land M, Mennie N, Rusted J (1999) The roles of vision and eye movements in the control of activities of daily living. Perception 28:1311-1328

McLeod P, Jenkins S (1991) Timing accuracy and decision time in high-speed ball games. Int J Sport Psychol 22:279-295

McLeod P, McLaughlin C, Nimmo-Smith I (1985) Information encapsulation and automaticity: evidence from the visual control of finely timed actions. In: Posner, Marin (eds) Attention and performance XI. Lawrence Erlbaum Associates, Hillsdale, pp 391-406

Müller H, Sternad D (2004) Decomposition of variability in the execution of goal-oriented tasks: three components of skill improvement. J Exp Psychol Hum Percept Perform 30:212-233

Regan D (1997) Visual factors in hitting and catching. J Sports Sci 15:533-558

Schmidt RA (1969) Movement time as a determiner of timing accuracy. J Exp Psychol 79:43-47

Tresilian JR, Plooy A (2006) Systematic changes in the duration and precision of interception in response to variation of amplitude and effector size. Exp Brain Res 171:421-435

Triesch J, Ballard DH, Hayhoe MM, Sullivan BT (2003) What you see is what you need. J Vision 3:86-94

Trommershauser J, Maloney LT, Landy MS (2003) Statistical decision theory and trade-offs in the control of motor response. Spat Vis 16:255-275

van Beers RJ (2007) The sources of variability in saccadic eye movements. J Neuroscience 27:8757-8770

van Beers RJ (2008) Saccadic eye movements minimize the consequences of motor noise. PLoS ONE 3(4):e2070

van Beers RJ, Sittig AC, Denier van der Gon JJ (1998) The precision of proprioceptive position sense. Exp Brain Res 122:367-377

van Beers RJ, Haggard P, Wolpert DM (2004) The role of execution noise in movement variability. J Neurophysiol 91:1050-1063

Virsu V, Oksanen-Hennah H, Vedenpää A, Jaatinen P, Lahti-Nuuttila $P$ (2008) Simultaneity learning in vision, audition, tactile sense and their cross-modal combinations. Exp Brain Res 186:525-537

Wallace SA, Weeks DL, Kelso JAS (1990) Temporal constraints in reaching and grasping behavior. Hum Mov Sci 9:69-93

Westheimer G, McKee SP (1977) Perception of temporal order in adjacent visual stimuli. Vis Res 17:887-892 